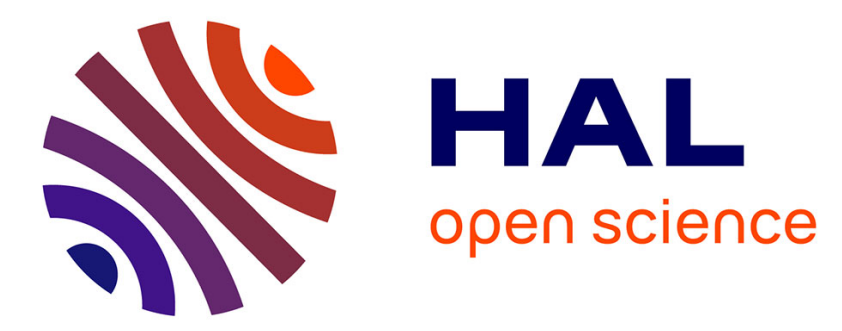

\title{
Modélisation de la propagation acoustique par la théorie des modes : application à la tomographie océanique P. Le Bras
}

\section{To cite this version:}

P. Le Bras. Modélisation de la propagation acoustique par la théorie des modes: application à la tomographie océanique. Journal de Physique IV Proceedings, 1994, 04 (C5), pp.C7-1083-C7-1086. 10.1051/jp4:19945238 . jpa-00252925

\section{HAL Id: jpa-00252925 https://hal.science/jpa-00252925}

Submitted on 1 Jan 1994

HAL is a multi-disciplinary open access archive for the deposit and dissemination of scientific research documents, whether they are published or not. The documents may come from teaching and research institutions in France or abroad, or from public or private research centers.
L'archive ouverte pluridisciplinaire HAL, est destinée au dépôt et à la diffusion de documents scientifiques de niveau recherche, publiés ou non, émanant des établissements d'enseignement et de recherche français ou étrangers, des laboratoires publics ou privés. 


\title{
Modélisation de la propagation acoustique par la théorie des modes : application à la tomographie océanique
}

\author{
P. LE BRAS
}

IFREMER Brest, Laboratoire d'Acoustique Sous-Marine, BP. 70, 29280 Plouzane, France

\begin{abstract}
Acoustic tomography monitors mesoscale fluctuations of ocean characteristics using prediction of rays travel times between sources and receivers. Unfortunately this method falls down near the channel axis because crowded rays cannot then be time resolved. We here first describe an implementation of a rangeindependant normal modes model to simulate acoustic propagation. Using the fact that crowded part of the time response can be described using only a small number of modes we then expose some results on modal filtering in the frequency domain applied to the analysis of this powerful ray-unresolved part of the response. We show that contribution of each mode to response can be pointed out using an approximate sound speed profile. We also compare results on the modal filtering with classical beamforming, showing the complementarity of these two array processings.
\end{abstract}

\section{CONTEXTE}

La tomographie $[1,2]$ est une méthode acoustique d'observation de l'océan à grande échelle qui consiste à mesurer les variations de la célérité du son autour d'un profil moyen connu dans la zone à étudier pour en déduire les champs de température, de vitesse et de vorticité. La zone à introspecter est maillée en un réseau d'émetteurs-récepteurs. L'identification, sur la réponse temporelle de chacun d'eux, de grandeurs significatives permet de remonter aux caractéristiques océanologiques des tranches d'eau verticales insonifiées par résolution du problème inverse.

Le modèle de propagation actuellement utilisé en tomographie, basé sur la théorie des rayons, porte naturellement les océanographes à identifier les temps d'arrivée des rayons propres. Cependant, quand on se rapproche de l'axe du chenal, les trajets deviennent de plus en plus nombreux et d'une densité temporelle incompatible avec une discrimination certaine. Ainsi, la partie la plus énergétique du signal et la plus riche en informations (l'essentiel des perturbations océaniques s'y trouvant concentré) reste inexploitée. L'insuffisance de la modélisation actuelle nous a amené à évaluer les possibilités qu'offre, pour la tomographie, une approche modale de la propagation, notamment dans l'étude de ces arrivées tardives.

\section{MODELISATION MODALE DE LA REPONSE TEMPORELEE:}

On se place dans une configuration où le profil de célérité ne dépend pas de la distance, et où l'amortissement en surface, dans l'eau et dans le fond est négligé. La bathycélérimétrie est décomposée en segments où le gradient de l'inverse du carré de l'indice de réfraction est supposé constant. Dans chaque strate la solution de l'équation de Green [3,4] s'exprime en termes de fonctions d'Airy. Par raccordement aux interfaces entre couches liquides de la pression et de la vitesse normale on explicite la solution générale de la fonction de Green sur toute la hauteur d'eau. La vérification des conditions aux limites, pression nulle en surface et raccordement à une onde évanescente dans le fond, donne les modes propagatifs relatifs à cette bathycélérimétrie pour une source monochromatique: $\phi_{m}(z)$. La sommation de la participation au champ de pression de tous les modes compose alors la fonction de transfert du milieu: $P(r, z, \omega)$.

Si l'on élargit ce calcul à un spectre de fréquences on peut, par l'utilisation de transformations de Fourier rapides, synthétiser la réponse temporelle d'un récepteur, $p(r, z, t)$, connaissant le spectre du signal source. 


$$
\begin{aligned}
P(r, z, \omega) & =\sum_{m} \phi_{m}(z) \phi_{m}\left(z_{\text {source }}\right) H_{o}\left(\zeta_{m} \cdot r\right) \\
p(r, z, t) & =\int_{-\infty}^{+\infty} P(r, z, \omega) S(\omega) e^{i \omega t} d \omega
\end{aligned}
$$

$H_{o}$ : fonction de Hankel;

$\omega:$ pulsation de la source monochromatique;

$S(\omega)$ : spectre de la source;

$(r, z)$ : distance, immersion du récepteur;

$\zeta_{m}$ : nombre d'onde horizontal du mode $m$.

Sur la réponse temporelle issue du calcul modal pour une configuration donnée (figure 1, bathycélérimétrie figure 2) on peut identifier des arrivées de rayons et, par là même, le comparer à un calcul de temps d'arrivée de rayons propres (points). L'adéquation est totale dans la zone où l'approximation géométrique, base de la théorie des rayons, est valable. De plus nous avons confronté, avec succès, les résultats de notre modèle à des calculs explicites menés pour des configurations simples (profil de célérité constant).

La synthèse de la réponse temporelle par les modes ne présente pas de différences marquantes par rapport à celle obtenue par les rayons pour les angles les plus inclinés. Cependant, il a été montré [5] que la partie finale dense en rayons contient un faible nombre de modes. Cela permet d'envisager la conception de nouvelles méthodes de traitement des signaux parallélement à l'émergence de nouveaux moyens de réception telle que l'antenne verticale: Le filtrage modal semble être une méthode d'analyse adéquate et pertinente pour cette partie trés énergétique de la réponse. Le logiciel de calcul modal nous permet d'évaluer les performances d'un tel traitement.

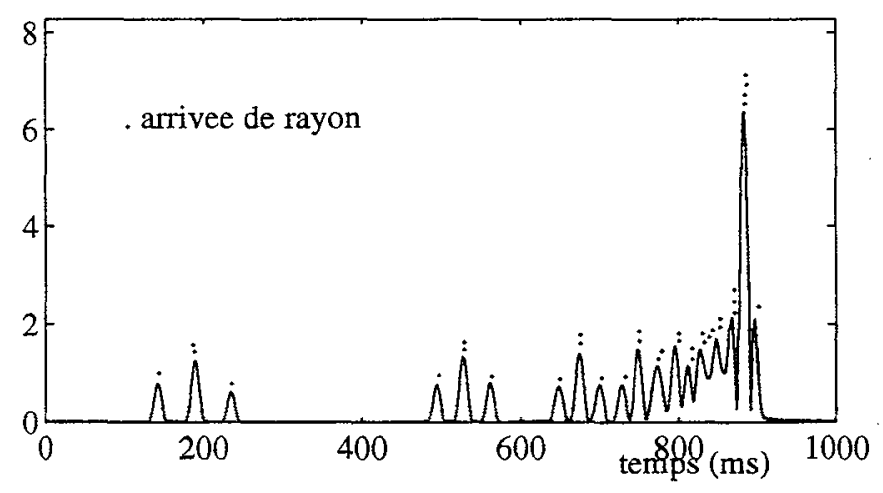

fig 1: Signal temporel au récepteur, distance: $126.685 \mathrm{~km}$ immersion source: $153 \mathrm{~m}$, immersion récepteur: $150 \mathrm{~m}$, signal: bande de $50 \mathrm{~Hz}$ centrée autour de $250 \mathrm{~Hz}$.

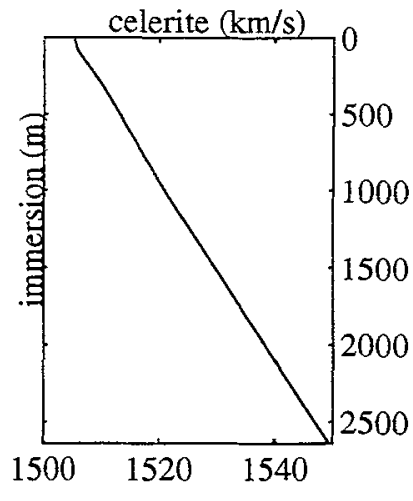

fig 2: bathycélérimétrie: Méditerranée hiver.

\section{FILTRAGE MODAL:}

Disposant d'une antenne verticale on peut pratiquer un filtrage modal. Le filtrage classique [7] s'effectue sur un signal monochromatique: il consiste à projeter le vecteur des réponses temporelles de l'antenne sur un vecteur de base, le mode que l'on désire filtrer. Conformément aux propriétés d'orthogonalité des modes, le résultat est alors la contribution de chacun d'eux à la réponse.

$$
f_{i l t_{m}}(r, t)=\int_{0}^{h e a u} \phi_{m}(z) \cdot p(r, z, t) d z
$$

filt $_{m}$ : résultat du filtrage suivant le mode $m$. heau: hauteur d'eau.

Cependant, dans le cas d'un signal de durée finie, ce traitement n'est valide que si la forme du mode varie peu en fonction de la fréquence, hypothèse incompatible avec la bande de fréquence utilisée pour les signaux de tomographie (fréquence porteuse de $250 \mathrm{~Hz}$, bande de $50 \mathrm{~Hz}$ ): ce type de filtrage n'est pas assez performant. On doit alors effectuer le filtrage dans le domaine fréquentiel. Les réponses temporelles de tous les récepteurs sont converties en spectres de fréquence par transformations de Fourier, le filtrage est effectué pour chaque fréquence du spectre puis reconvertie en réponse temporelle par transformée de Fourier inverse. On retrouve, à un coefficient multiplicateur près, la contribution à la réponse totale du mode filtré. Ce traitement met en évidence le caractère dispersif de la propagation: chaque mode est caractérisé par sa courbe de dispersion [6] 
donnant la vitesse de groupe (vitesse de propagation de l'énergie contenue dans le mode) en fonction de la fréquence (figure 3). L'etalement temporel du mode nous permet, connaissant la distance de propagation, d'estimer la vitesse de groupe moyenne de chaque mode filtré, grandeur caractéristique nécessaire à la résolution du problème inverse.

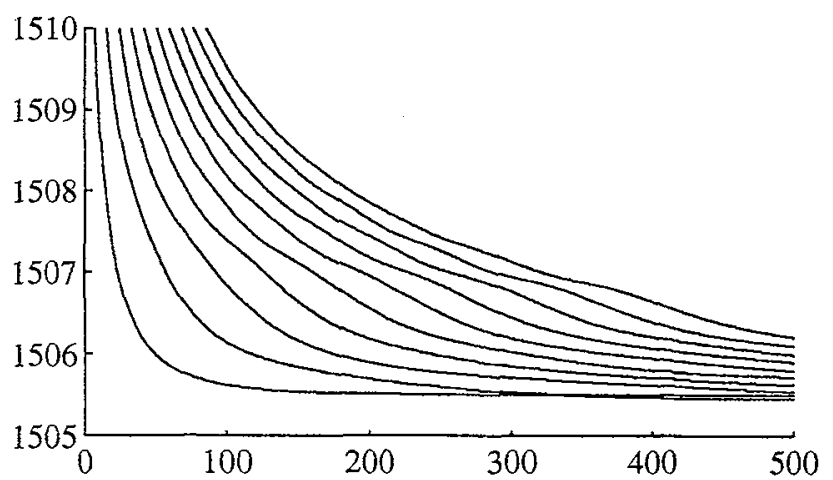

fig 3: vitesse de groupe des 10 premiers modes relatifs au profil de la figure 2.

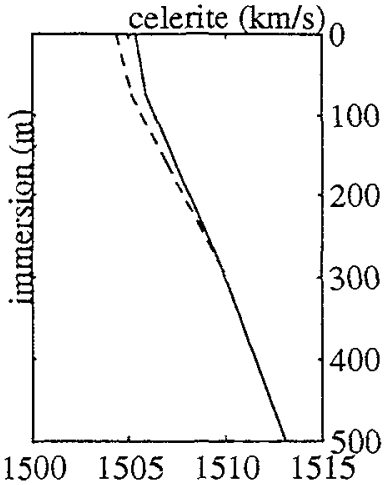

fig 4: aétail du profil exact (trait plein) et du profil dégradé (pointillé).

Ce traitement idéal suppose la connaissance parfaite du profil de célérité au récepteur afin de déterminer les fonctions de base du filtre. Or ce profil n'est connu qu'approximativement (profil moyen). Cependant, on montre, par des simulations numériques, que l'on peut appliquer ce traitement en filtrant le signal reçu suivant des modes calculés pour une bathycélérimétrie approchée.

\section{SIMULATIONS NUMERIQUES:}

Les réponses temporelles de l'antenne sont calculées pour un profil de célérité du type Méditerranée hiver (figure 2). L'antenne est déployée de la surface jusqu'à la profondeur de 1000 mètres, contenant 200 récepteurs équirépartis. La distance entre la source et l'antenne est de 126.685 kilométres. Le signal source a une bande de fréquence de $50 \mathrm{~Hz}$ centrée autour de $250 \mathrm{~Hz}$. L'émetteur est à une profondeur de 153 mètres. Dans un premier temps on applique le filtrage idéal (même profil pour le calcul des réponses et des fonctions de base du filtre) pour les 30 premiers modes. On retrouve alors exactement les contributions de chacun d'eux à la réponse temporelle (figure 5). On poursuit la simulation (figure 6) en dégradant le profil bathycélérimétrique (figure 4) utilisé pour le calcul des fonctions de base du filtre. On constate, par superposition des deux images, que malgré la désadaptation entres les modes filtrés et ceux du filtre on retrouve le résultat du filtrage idéal (figure 5) fort peu dégradé pour les modes d'ordre faible. Par contre le résultat se dégrade quand l'ordre du mode augmente.

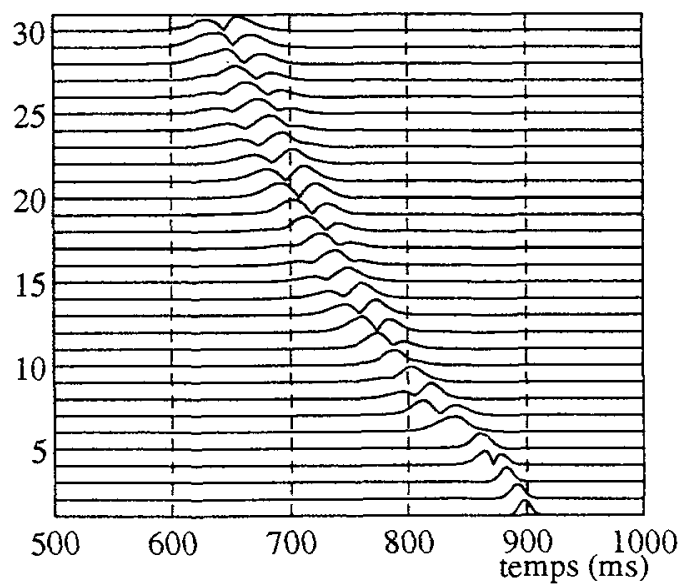

fig 5: résultat du filtrage idéal.

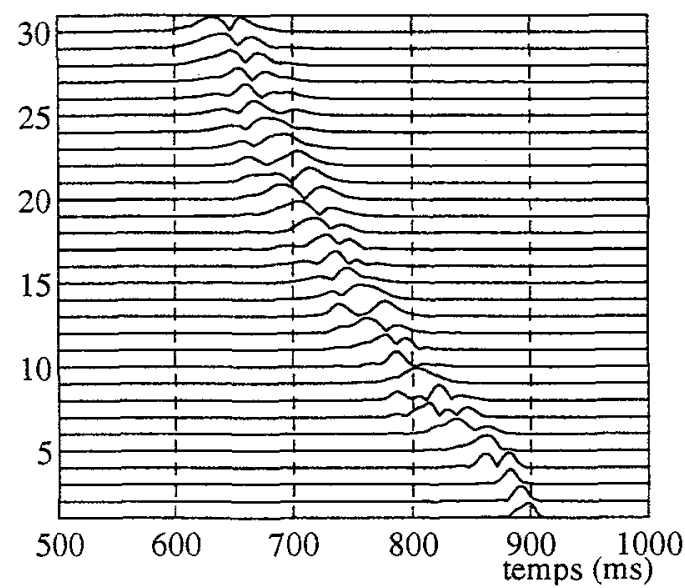

fig 6: résultat du filtrage dégradé. 
Parallèlement on effectue un traitement classique d'antenne par formation de voies (figure 7 ) sur lequel on peut identifier avec sûreté les angles et temps d'arrivée des rayons propres jusqu'à une certaine limite temporelle au delà de laquelle ils ne sont plus discriminables (environ $750 \mathrm{~ms}$ pour cet exemple): l'hypothèse de l'onde plane s'écroule et le champ n'est plus homogène sur toute la hauteur de l'antenne. Par opposition (figure 5), les modes sont assez finement définis pour être utilisables dans la zone dense en rayons, par contre sont trop étalés temporellement et trop mal filtrés pour être significatifs avant $700 \mathrm{~ms}$. Ceci met en évidence la complémentarité des deux approches: les rayons pour les premières arrivées et les modes pour décrire la structure tardive de la réponse temporelle. Lintérêt de l'analyse modale est spécialement évident dans la tranche [ $800-900 \mathrm{~ms}]$ de l'exemple présenté ici.

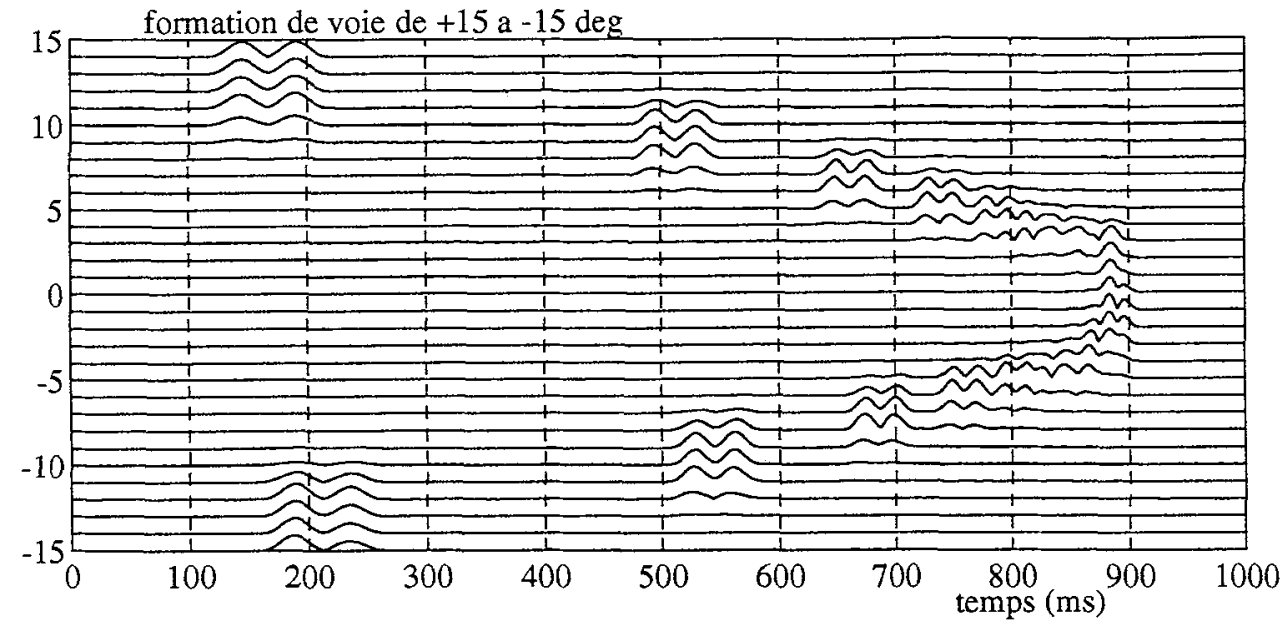

fig 6: résultat du traitement d'antenne par formation de voies: hauteur antenne: $300 \mathrm{~m}$, immersion centre: $150 \mathrm{~m}$, distance: $126.685 \mathrm{~km}$, bathycélérimétrie: figure 2.

\section{CONCLUSIONS:}

On peut, à partir d'une bathycélérimétrie approchée, extraire des réponses temporelles d'une antenne la contribution de chaque mode et par là même identifier les vitesses de groupe de chacun d'eux, grandeurs caractéristiques permettant la résolution du problème inverse. Il reste, à ce stade, à pousser plus avant l'étude de la robustesse d'un tel traitement, notamment la quantification de l'influence de paramètres tel que: dimension de l'antenne, erreurs de positionnement et de verticalité, bruit de fond, dégradation du signal au cours des traitements, nature du signal source, différence entre les bathycélérimétries moyenne et réelle... L'objectif visé est l'élaboration d'une méthode globale d'analyse du signal basée sur la complémentarité du filtrage modal et de la formation de voies.

\section{REFERENCES:}

Périodique: [1] Munk W.H et C.Wunsh: Ocean acoustic tomography: Ascheme for large scale monitoring. Deep sea res., 26A, 123-160, 1979.

Périodique: [2] Spindel R.C et P.F. Worcester: Ocean acoustic tomography: A decade of development. Sea technology, july 1991.

Livre: [3] Brekhovskikh L. et Y. Lysanov: Fundamentals of ocean acoustics. Springer series in electrophysics 8, Springer-Verlag, 1982.

Livre: [4] Tolstoy I. et C.S. Clay: Ocean acoustic: Theory and experiment in underwater sound. Am. Inst. of physics, $381 \mathrm{p}, 1987$.

Périodique: [5] Munk W.H et C.Wunsh: Ocean acoustic tomography: Rays and modes. Rev. of geophysics and space physics, vol 21, no 4, 777-793, 1983.

Périodique: [6] Porter R.P.: Dispersion of axial SOFAR propagation in the western Mediterranean. J. Acoust. Soc. Am. 53, 181-191, 1973.

Thèse: [7] Sessarego J.P: Filtrage spatial des modes par petits fonds marins. Rapport de thèse présenté à la faculté des sciences de l'université de Provence, Janv 1977. 